\title{
Frequency of occurrence as a psychophysical continuum: Weber's fraction, Ekman's fraction, range effects, and the phi-gamma hypothesis
}

\author{
DAVID C. RUBIN \\ Lawrence University, Appleton, Wisconsin 54911
}

\begin{abstract}
Using the continuum of frequency of occurrence of words in English, it was found that: (1) errors in judgment are distributed lognormally rather than normally, and therefore the standard method of calculating Weber's fraction underestimates its definition, (2) Weber's fraction has an extremely large value of 3.3, (3) Ekman's fraction equals 1.81, not .03 as with sensory continua, and (4) the logarithm of the dynamic range times Stevens' law exponent equals 3.83 , not 1.53 as with sensory continua. The last two results favor Teghtsoonian's underlying sensory scale interpretation over Poulton's range effects interpretation of the range-exponent relation.
\end{abstract}

The continuum of frequency of occurrence of words in English has an extremely large Weber's fraction, $\Delta \phi / \phi$ (Underwood, 1966), and thus lends itself to the study of two unresolved issues in psychophysics. The first issue is whether errors in judgment on a prothetic continuum are distributed normally, as is commonly assumed, or whether they are distributed lognormally, as Stevens (1975) claims. The second issue is whether Ekman's fraction, $\Delta \psi / \psi$, is a constant for all continua (Teghtsoonian, 1971, 1974).

\section{THE PHI-LOG-GAMMA HYPOTHESIS AND WEBER'S FRACTION}

A common assumption of indirect scaling in general, and the method of constant stimuli in particular, is that errors of judgment are distributed normally with respect to the physical stimulus. In an elegant theoretical paper, Thurstone (1928), however, demonstrated that this assumption, known as the phi-gamma hypothesis, is not consistent with Weber's law. In fact, the phi-gamma hypothesis can only hold if $\Delta \phi$, the jnd, does not vary with $\phi$ : that is, if $\Delta \phi=C$. When Weber's law holds, errors are distributed normally with respect to the logarithm of the physical stimulus: that is, the phi-log-gamma hypothesis holds. In general, the distribution of errors must be consistent with the function relating to $\Delta \phi$ to $\phi$. As Weber's law is a good approximation for prothetic continua, the phi-log-gamma hypothesis should also be a good approximation for prothetic continua.

The phi-log-gamma hypothesis has been shown to have a strong theoretical, but only a marginal

I wish to thank Benton J. Underwood for making this data available to me. empirical, advantage over the phi-gamma hypothesis (Engen, 1972; Guilford, 1931). In order to obtain a clear empirical comparison of the two hypotheses, measurements must be made over a range where a logarithmic and a linear scale differ significantly. If Weber's fraction is small, so that the range of comparison stimuli is small, and if, because of thresholds, measurements must be made away from $\phi=0$, then a logarithmic and a linear scale provide good approximations to each other and differences are hard to detect. For the continuum of frequency of occurrence of words in English, however, Weber's fraction is large and the range can include points near $\phi=0$, making it a convenient continuum for an empirical comparison.

As an example of the method of constant stimuli, Underwood (1966, pp. 177-179) had 50 subjects judge which word of each of 64 pairs of words was more frequent. The standard stimulus frequency was approximately 310 , and the eight comparison stimulus frequencies were logarithmically spaced over the range from approximately 1 to 75,000 . There were eight words at each frequency. Reanalysis of his data shows that the $z$-scores corresponding to the probability of a comparison stimulus being judged more frequent correlate more highly with the logarithm of the frequency than with the frequency itself $[r=.965$ vs. $r=.652, t(5)=2.64$, $p<.05]$, an outcome that supports the phi-loggamma hypothesis. The results of this analysis are presented in Figure 1. Both the best fitting line from the phi-log-gamma and the phi-gamma hypotheses are shown.

The phi-log-gamma hypothesis implies that the usual method of calculating $\Delta \phi$, the jnd in Weber's fraction, results in an underestimate. $A$ jnd is defined as the change in the physical stimulus which is just noticeably $50 \%$ greater than chance, or $\phi .75-\phi .50$ in 


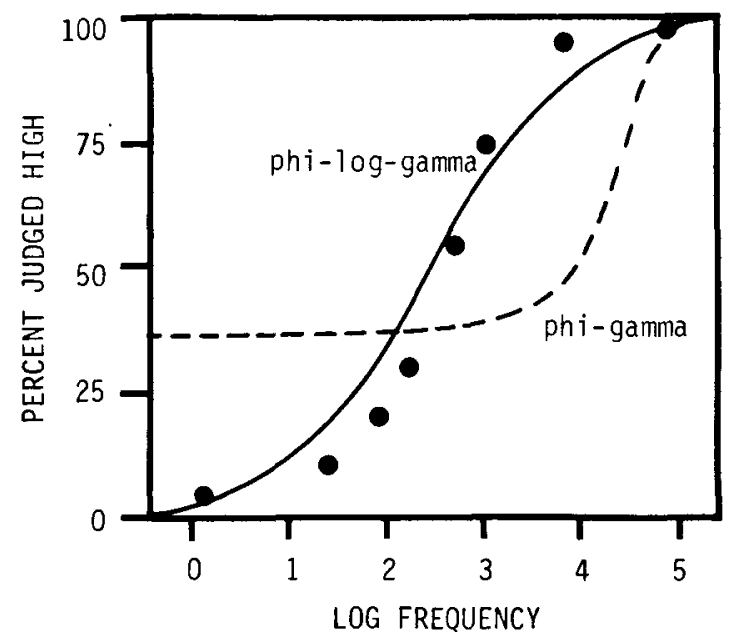

Figure 1. Percent of comparison stimuli judged more frequent than the standard.

the method of constant stimuli. The usual method of calculating a jnd, however, is $(\phi .75-\phi .25) / 2$. If the phi-gamma hypothesis holds, these two expressions would be equivalent. If the phi-log-gamma hypothesis holds, they would be different. In fact, if Weber's fraction as defined equals $C$, then under the phi-log-gamma hypothesis Weber's fraction as calculated would equal $\mathrm{C}-\mathrm{C}^{2} / 2(1+\mathrm{C})$, and thus would underestimate its definition by $\mathrm{C}^{2} / 2(1+C)$. For Underwood's (1966) frequency of occurrence data, where $C=4.7$, the underestimate would be $41 \%$. For the largest measured value for a sensory continuum, where $\mathrm{C}=.25$ (odor intensity; Engen, 1972), the underestimate would be $10 \%$. For a more typical value of $\mathrm{C}=.02$ the underestimate would be $1 \%$. Even an error of $1 \%$, however, is large enough to be important where jnds are summed to form a Fechnerian scale.

\section{RANGE EFFECTS AND EKMAN'S FRACTION}

Teghtsoonian (1971) has proposed that for any sensory continuum, the exponent in Steven's law is given by $1.53 / \log$ (dynamic range). On the basis of this and earlier calculations, Poulton (1968, 1975) has claimed that the value of the exponent in Stevens' law can be explained simply as a range effect. Teghtsoonian (1971), however, provides a different interpretation. He derives the range-exponent relation from Stevens' law and from the assumption that there is only one range of sensory magnitude into which all sensory judgments are made. To support this assumption he hypothesized that if there is only one range of sensory magnitude, then there should be only one value of Ekman's fraction, $\Delta \psi / \psi$, for that scale, just as there is only one value of Weber's fraction, $\Delta \phi / \phi$, for each physical scale. Although the exponent, $n$, in Stevens' law and Weber's fraction, $\Delta \phi / \phi$, varies greatly from continuum to continuum, Ekman's fraction, which is calculated from these two values by the formula $(1+\Delta \phi / \phi)^{\mathrm{n}}-1$, is approximately .03 for all continua tested (Teghtsoonian, 1971).

Initially, Poulton (1968) and Teghtsoonian (1971) disagreed on whether the important range was the actual range experienced by judges in an experiment or the range experienced by judges in their lifetime. Teghtsoonian (1973) demonstrated that both ranges have an effect, but that experimentally produced intramodal variation in range cannot produce changes in the exponent as large as those associated with intermodal variation in dynamic range. Poulton (1975) agreed, but did not change his interpretation that range effects are an artifact that determine the exponent in the power law.

Thus, the present status of the Poulton-Teghtsoonian debate is that both sides agree that intermodal range is the main cause of the range-exponent relation and thus, the two opposing interpretations are consistent with all existing data. Frequency of occurrence, however, provides data that is not consistent with both interpretations.

Consider what the two opposing interpretations would predict for the nonsensory continuum of frequency of occurrence of words in English. Teghtsoonian's interpretation is neutral. Frequency of occurrence may or may not be judged on the same subjective scale as sensory continua. From Poulton's range effect interpretation, however, there is no basis for predicting differences between frequency of occurrence and the sensory continua already studied, and the range-exponent relation should hold.

The value of the power law exponent for frequency of occurrence in English for groups of subjects varies between .51 and .92 (Carroll 1971; Shapiro, 1969). Substituting a representative value of .70 in the range-exponent relation, the range for frequency of occurrence should be 150 . The actual range of stimuli in the experiment which provided the .70 value was 300,000 . If the range experience by subjects in real life and not just in experiments is considered (Teghtsoonian, 1973), the discrepancy would be much greater. Thus, existing frequency of occurrence data goes against the range effect interpretation of the range-exponent relation.

To be consistent with Teghtsoonian's interpretation, one other criterion should be met. The value of Ekman's fraction should, except by coincidence, be different from the .03 value obtained for sensory continua. Fortunately, Underwood (1966) not only provides us with the data needed to calculate Weber's fraction for three-letter words, but also the data needed to calculate the power law exponent and thus Ekman's fraction. According to his data, the power law exponent for three-letter words is .34, yielding an Ekman's fraction of .81. This value is over 20 times greater than any yet reported (Teghtsoonian, 1974). 
Thus, while it appears that for sensory continua just noticeable differences occur when sensory magnitudes are altered by the same constant fraction, that fraction does not hold for all nonsensory continua. This finding supports the hypothesis that frequency of occurrence is not judged on the same subjective scale as sensory continua.

\section{EXPERIMENTAL DETERMINATION OF WEBER'S FRACTION FOR FREQUENCY OF OCCURRENCE}

Underwood's (1966) measurement of Weber's fraction for frequency of occurrence was replicated with two modifications. First, the comparison stimuli were placed at equal intervals along the frequency scale to insure that Underwood's (1966) procedure of placing them at equal ratios did not bias his results towards the phi-log-gamma hypothesis. Second, words of various letter lengths were sampled from the Kučera and Francis (1967) word count, so that a value of Weber's fractions for all words and not just three-letter words could be obtained. This value can be used with existing measurements of the power law exponent to calculate Ekman's fraction.

\section{METHOD}

Subjects. Twelve undergraduates took part in the experiment. Materials. Six comparison stimulus frequencies $(1,34,67,133$, 166 , and 199 per million) were evenly spaced above and below a standard stimulus frequency of 100 per million. There were 16 words at each comparison stimulus frequency and $96(6 \times 16)$ words at the standard stimulus frequency. The words were chosen from the Kučera and Francis (1967) word count. When there were not enough words at a particular frequency, words with the next higher and lower frequency were added. When there was an excess of words at a given frequency, words were selected randomly. Contractions were excluded. Each word was printed on a $3 \times 5$ in. index card.

Procedure. Following Underwood (1966), a forced choice constant stimulus method (Guilford, 1954) was used. The order of presentation of the 96 standard and the 96 comparison stimulus cards was random. The subject's task was to indicate whether the comparison stimulus was more or less frequent than the standard stimulus with which it happened to be paired. Subjects indicated a response by placing the comparison stimulus into an area labeled either "more frequent" or "less frequent." The subjects were instructed to judge only on the basis of the frequency of the particular orthographic form shown. The task was repeated until all 96 comparison stimulus words were judged.

\section{Results}

The probabilities of subjects judging words with frequencies $1,34,67,133,166$, and 199 occurrences per million as being more frequent than words with a frequency of 100 occurrences per million are: .03 , $\mathrm{SD}=.05 ; .45, \mathrm{SD}=.12 ; .54, \mathrm{SD}=.05 ; .56$, $\mathrm{SD}=.18 ; .59, \mathrm{SD}=.12 ;$ and $.75, \mathrm{SD}=.11$, respectively. Under the phi-gamma hypothesis, the best fitting line is $z=.009 \phi-1.089, r=.795$. Under the phi-log-gamma hypothesis, the best fitting line, $z=1.045 \log (\phi)-1.895$, has a significantly greater correlation coefficient of $.984[\mathrm{t}(3)=3.41$, $p<.05]$. The value of Weber's fraction calculated from the logarithmic fit is 3.3.

Using the .70 value of Stevens' law exponent for frequency of occurrence in English and the value of Weber's fraction obtained here, Ekman's fraction is 1.81 . This is 60 times greater than the average value of .03 found by Teghtsoonian for sensory continua. While the value of the Stevens' law exponent may be an overestimate of the true value due to the restricted range of stimuli afforded by word counts (Teghtsoonian, 1973), it is doubtful that an increased range would lower the exponent from .70 to the value of .02 needed to obtain an Ekman's fraction of .03 . Likewise, it is doubtful that the vaiue of Weber's fraction could be made small enough to yield a .03 Ekman's fraction.

\section{DISCUSSION}

The methods of psychophysics can be applied to a great variety of nonsensory continua (Stevens, 1975), and frequency of occurrence is one that has received a good deal of attention (Rubin, 1976). There is, however, no reason for every application of psychophysical methods to capture all the regularities found with sensory continua. If this were, in fact, the case, it might appear that the regularities found with sensory continua were a product of the methods used and not the sensory systems studied.

In particular, Teghtsoonian's claim that there is one underlying scale of subjective judgment cannot be extended to all continua. Likewise, Poulton's claim that the exponent in Stevens' law is determined by the artifact of dynamic range cannot be extended to all continua. The implications for the two claims are, however, quite different. If range effects are a valid explanation of the power law exponent, they must predict all exponents. For Teghtsoonian's claim this is not the case. The greatly different value of 1.81 for Ekman's fraction obtained here for a nonsensory continuum accentuates the small variation of .026 to .034 obtained for sensory continua.

Recently, Teghtsoonian (1974) has reformulated his position in terms of only observables, and thus without reference to subjective sensory scales or Ekman's fraction. Instead of proposing that Ekman's fraction is the same for all sensory continua, he proposes that jnds are equivalent across continua. That is, if two stimuli on different continua are judged to be equally intense, then other stimuli on those two continua which are equal numbers of jnds away from the first two will also be judged equally intense (Auerbach, 1971). Under this view, Ekman's fraction can be seen as Weber's fraction for the number scale. The implications of the results found here do not change significantly. Instead of limiting the constant .03 value of Ekman's fraction to sensory continua, they limit the assumption of the equivalence of $\mathrm{jnds}$ to sensory continua. 


\section{REFERENCES}

Auerbach, C. Interdependence of Stevens' exponents and discriminability measures. Psychological Review, 1971, 78, 556.

CARRol. , J. B. Measurement properties of subjective magnitude estimates of word frequency. Journal of Verbal Learning and Verbal Behavior, 1971, 10, 722-729.

Engen, T. Psychophysics: I. Discrimination and detection. In J. W. Kling and L. A. Riggs (Eds.), Experimental psychology (Vol. I) Sensation and perception. New York: Holt, Rinehart, and Winston, 1972.

Guilford. J. P. Some empirical tests of the method of paired comparisons. Journal of General Psychology, 1931, 5, 64-77.

GuILFORD, J. P. Psychometric methods. New York: McGraw-Hill, 1954.

KuĆERA, H., \& FRANCIS, W. N. Computational analysis of present-day American English. Providence, R.I: Brown University Press, 1967.

Poulton, E. C. The new psychophysics: Six models for magnitude estimation. Psychological Bulletin, 1968, 69, 1-19.

Poulton, E. C. Range effects in experiments on people. American Journal of Psychology, 1975, 88, 3-32.

RubiN. D. C. Applying psychometric methods in linguistic research: Some recent advances. Linguistics, 1976, 168, 63-66.

SHAPIRO, B. J. The subjective estimation of relative word frequency. Journal of Verbal Learning and Verbal Behavior. $1969,8,248-251$

Stevens, S. S. Psychophysics. New York: Wiley, 1975.

Tegrtsoonian, R. On the exponent in Stevens' law and the constant in Ekman's law. Psychological Review, 1971, 78, 71-80.

Teghtsoonian, R. Range effects in psychological scaling and a revision of Stevens' law. American Journal of Psychology, 1973 , 86. 3-27.

Teghtsoonian, R. On facts and theories in psychophysics: Does Ekman's law exist? In H. R. Moskowitz, J. C. Stevens, \& B. Scharf (Eds.), Sensation and measurement: Papers in honor of S. S. Stevens. Dordrecht, Holland: Reidel, 1974.

ThuRstone, L. L. The phi-gamma hypothesis. Journal of Experimental Psychology, 1928, 11, 293-305.

UNDERWOOD, B. J. Experimental psychology. New York: Appleton-Century-Crofts, 1966.

(Received for publication April 2, 1976; revision received July 13,1976 .) 